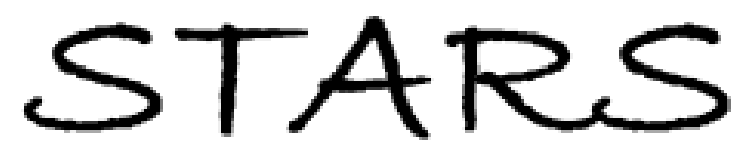

University of Central Florida

STARS

$1-1-2010$

\title{
Extended Kerr effect of polymer-stabilized blue-phase liquid crystals
}

Jin Yan

University of Central Florida

Hui-Chuan Cheng

University of Central Florida

Sebastian Gauza

University of Central Florida

Yan Li

University of Central Florida

Meizi Jiao

University of Central Florida

Find similar works at: https://stars.library.ucf.edu/facultybib2010

University of Central Florida Libraries http://library.ucf.edu

See next page for additional authors

This Article is brought to you for free and open access by the Faculty Bibliography at STARS. It has been accepted for inclusion in Faculty Bibliography 2010s by an authorized administrator of STARS. For more information, please contact STARS@ucf.edu.

\section{Recommended Citation}

Yan, Jin; Cheng, Hui-Chuan; Gauza, Sebastian; Li, Yan; Jiao, Meizi; Rao, Linghui; and Wu, Shin-Tson, "Extended Kerr effect of polymer-stabilized blue-phase liquid crystals" (2010). Faculty Bibliography 2010 s. 967.

https://stars.library.ucf.edu/facultybib2010/967

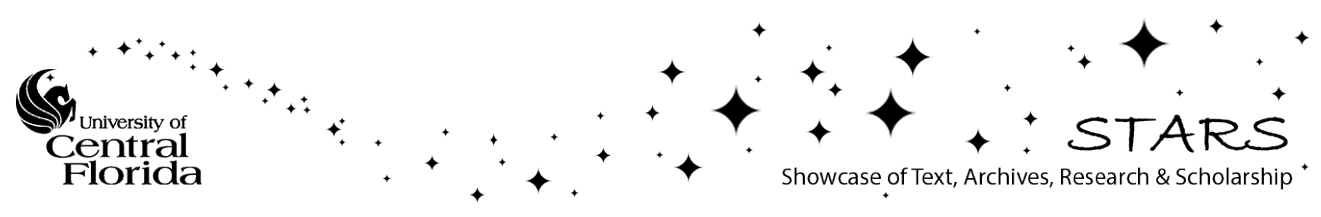


Authors

Jin Yan, Hui-Chuan Cheng, Sebastian Gauza, Yan Li, Meizi Jiao, Linghui Rao, and Shin-Tson Wu 


\section{Extended Kerr effect of polymer-stabilized blue-phase liquid crystals}

Cite as: Appl. Phys. Lett. 96, 071105 (2010); https://doi.org/10.1063/1.3318288

Submitted: 24 November 2009 . Accepted: 23 January 2010 . Published Online: 17 February 2010

Jin Yan, Hui-Chuan Cheng, Sebastian Gauza, Yan Li, Meizi Jiao, Linghui Rao, and Shin-Tson Wu

\section{ARTICLES YOU MAY BE INTERESTED IN}

Electro-optics of polymer-stabilized blue phase liquid crystal displays

Applied Physics Letters 94, 101104 (2009); https://doi.org/10.1063/1.3097355

A large Kerr constant polymer-stabilized blue phase liquid crystal

Applied Physics Letters 98, 081109 (2011); https://doi.org/10.1063/1.3559614

Low voltage blue-phase liquid crystal displays

Applied Physics Letters 95, 231101 (2009); https://doi.org/10.1063/1.3271771

\section{Applied Physics Reviews} Now accepting original research 


\title{
Extended Kerr effect of polymer-stabilized blue-phase liquid crystals
}

\author{
Jin Yan, Hui-Chuan Cheng, Sebastian Gauza, Yan Li, Meizi Jiao, Linghui Rao, and \\ Shin-Tson $\mathrm{Wu}^{\mathrm{a}}$ \\ College of Optics and Photonics, University of Central Florida, Orlando, Florida 32816, USA
}

(Received 24 November 2009; accepted 23 January 2010; published online 17 February 2010)

\begin{abstract}
Electric-field-induced birefringence of a polymer-stabilized blue-phase liquid crystal (BPLC) is investigated. In the low field region, conventional Kerr effect holds. As the electric field increases, the induced birefringence gradually saturates and deviates from Kerr effect. An exponential convergence model, called extended Kerr effect, is proposed to fit the experimental data. Good agreement between experiment and model is obtained. This extended Kerr effect will make a significant impact to the optimization of emerging BPLC display devices. (C) 2010 American Institute of Physics. [doi:10.1063/1.3318288]
\end{abstract}

Polymer-stabilized blue-phase liquid crystal (PS-BPLC) (Refs. 1-7) is emerging as next-generation display technology because it exhibits some revolutionary features, such as no need for a surface alignment layer, submillisecond grayto-gray response time, ${ }^{8}$ and inherently wide viewing angle. The underlying physical mechanism of PS-BPLC is electricfield-induced birefringence known as Kerr effect. ${ }^{9}$ In the Kerr effect the induced birefringence is linearly proportional to $E^{2}$, where $E$ is the electric field intensity. However, this linear relationship is valid only in the low field region. As $E$ increases, the induced birefringence cannot increase indefinitely, i.e., it will gradually saturate. Such a saturation phenomenon has been observed in polar solutions several decades ago, ${ }^{10}$ however it remains unexplored in PS-BPLC composite systems. Therefore, there is an urgent need to discover a simple convergence form in order to accurately model the display performances.

In this letter, we report the measured induced birefringence of a BPLC cell and then fit the experimental data with Kerr effect and our proposed convergence model, called extended Kerr effect. As expected, Kerr effect is valid only in the low field region, while the extended Kerr effect works very well in the entire region. Based on this extended Kerr effect we further fit the measured voltage-dependent transmittance (VT) curve of a PS-BPLC display device with inplane switching (IPS) electrodes. ${ }^{11}$ Again, good agreement between model and experiment is found.

The PS-BPLC employed in this study is a mixture consisting of nematic LC (49 wt\% Merck BL038), chiral dopants (21\% Merck CB15 and 6\% ZLI-4572) and monomers (9\% EHA and 15\% RM257). The BPLC was sandwiched between two indium-tin-oxide glass substrates with a cell gap of $8 \mu \mathrm{m}$. To investigate its electro-optical properties, we used Michelson interferometer as depicted in Fig. 1 in order to double the fringe shift because of double pass. The light source was an unpolarized He-Ne laser $(\lambda=633 \mathrm{~nm})$. The laser beam was split equally into two arms by the beam splitter (BS). The BPLC cell was placed in one arm and was driven by a square-wave voltage with $1 \mathrm{kHz}$ frequency. Fringes were observed after the objective lens and an iris was placed in front of the photodiode detector (D). Through the measured light intensity, we can obtain the induced birefrin-

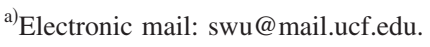

gence. The whole system was built on a floating optical table to minimize any environment-induced fluctuation. Figure 2 depicts the measured refractive index change $(\delta n)$ of the BPLC cell under different voltages.

In a voltage-off state, the BPLC is optically isotropic. Its refractive index can be written as follows: ${ }^{12}$

$$
n_{\text {iso }}=\sqrt{\left(2 n_{o}^{2}+n_{e}^{2}\right) / 3},
$$

where $n_{o}$ and $n_{e}$ are the ordinary and extraordinary refractive indices of the LC composite, respectively. When the birefringence is small, Eq. (1) can be approximated by:

$$
n_{i s o} \approx\left(2 n_{o}+n_{e}\right) / 3 \text {. }
$$

To validate this approximation, let us assume the LC composite has $n_{o}=1.50$ and $n_{e}=1.70$. The difference between Eqs. (1) and (2) is only $\sim 0.2 \%$. When an electric field is applied, birefringence is induced. The optic axis of the induced refractive-index ellipsoid is along the electric field direction. For the PS-BPLC, the induced birefringence is due to the local reorientation of the molecules; ${ }^{1}$ therefore, it is quite reasonable to assume that the average refractive index keeps constant at any electric field

$$
n_{\text {average }}=\frac{2 n_{o}(E)+n_{e}(E)}{3}=n_{i s o} .
$$

Here, $n_{o}(E)$ and $n_{e}(E)$ are the field dependent refractive index perpendicular and parallel to the electric field, respectively. With this assumption, the refractive index change un-

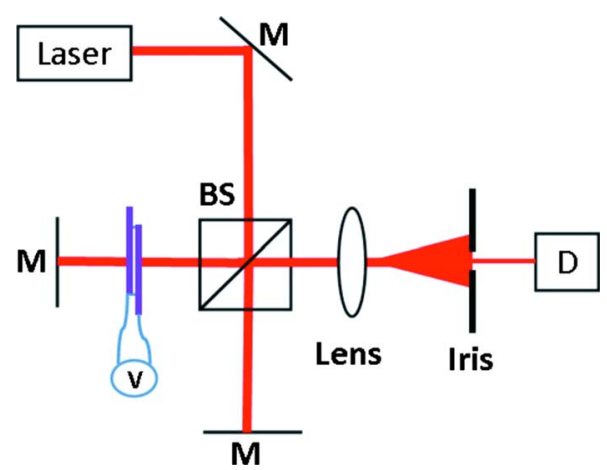

FIG. 1. (Color online) Experimental setup of Michelson interferometer for measuring the refractive index change of a PS-BPLC cell. M: mirror, BS: beam splitter, and D: detector. 


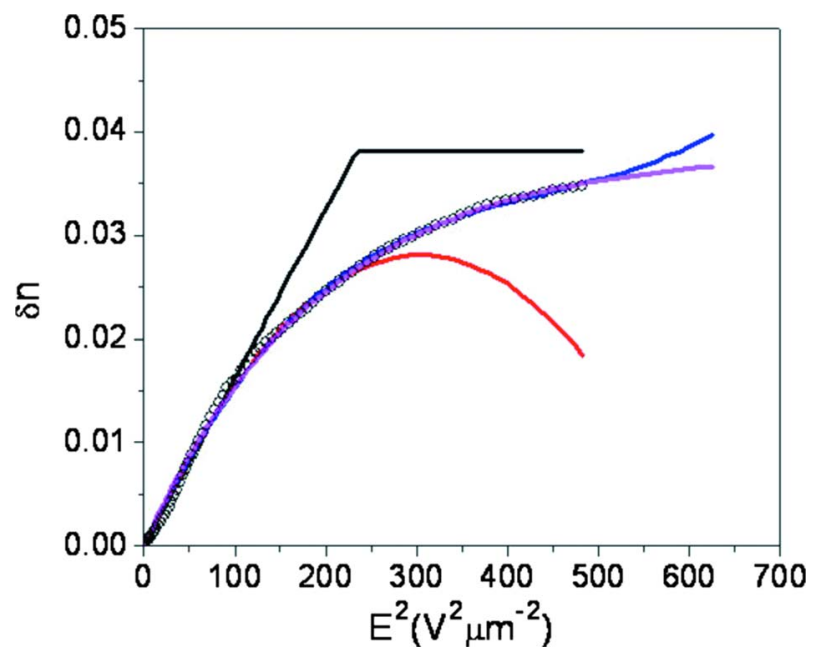

FIG. 2. (Color online) Measured refractive index change (circles) and fittings with Eq. (6) (black straight lines), Eq. (7) (red parabolic lines), the model including second, fourth, and sixth order terms (blue upward lines), and the extended Kerr effect Eq. (8) (magenta convergent lines).

der an electric field can be expressed as follows:

$$
\delta n=n_{\text {iso }}-n_{o}(E)=\frac{n_{e}(E)-n_{o}(E)}{3} .
$$

Equation (4) correlates the refractive index change we measured with the induced birefringence. The induced birefringence $\left(\Delta n_{\text {ind }}\right)$ of PS-BPLC under an external electric field is governed by Kerr effect as follows: ${ }^{9}$

$$
\Delta n_{\text {ind }}=n_{e}(E)-n_{o}(E)=\lambda K E^{2} .
$$

Here, $\lambda$ is the wavelength, $K$ is the Kerr constant, and $E$ is the amplitude of the electric field. Equation (5) is valid only in the low electric field region; otherwise, it leads to a divergence as $E$ keeps increasing. Physically, the induced birefringence cannot exceed the intrinsic effective birefringence of the BPLC composite when all the LC directors are reoriented by the strong electric field.

To avoid divergence of the induced birefringence in high field region, a saturation field $\left(E_{s}\right)$ has been introduced and Eq. (5) modified as follows: ${ }^{4,5}$

$$
\Delta n_{\text {ind }}=(\Delta n)_{o}\left(E / E_{s}\right)^{2},
$$

where $(\Delta n)_{o}$ denotes the maximum induced birefringence of the LC composite. For the convenience of discussion, let us refer Eq. (6) as truncation model. In the truncation model, the induced birefringence is described as $\Delta n_{\text {ind }}$ $=(\Delta n)_{o}\left(E / E_{s}\right)^{2}$ when $E<E_{s}$ and $\Delta n_{\text {ind }}=(\Delta n)_{o}$ when $E \geq E_{s}$. Such an abrupt change in the induced birefringence serves the purpose to prevent divergence, but in reality as the electric field increases the induced birefringence will gradually saturate because all the LC directors will be eventually reoriented by the electric field.

In Fig. 2, the measured refractive index change is linearly proportional to $E^{2}$ as expected from Kerr effect in the weak field region. As the electric field increases, the induced refractive index change $(\delta n)$ gradually saturates. To explain this trend, higher order electro-optical effects have been considered. ${ }^{13}$ For a centrosymmetric crystal, the odd-order terms vanish due to the inversion symmetry, and only the even-order terms remain. Including the fourth-order term, the refractive index change can be written as follows:

$$
\delta n=\frac{\lambda\left(K_{1} E^{2}+K_{2} E^{4}\right)}{3},
$$

where $K_{1}$ and $K_{2}$ are the Kerr constant and fourth-order coefficient, respectively. Equation (7) is used to fit the experimental data with $K_{1}$ and $K_{2}$ as adjustable parameters. As depicted in Fig. 2, the fitting (red curve) is good when $E^{2}$ is below $200 \mathrm{~V}^{2} / \mu \mathrm{m}^{2}$. However, above this field the fourth-order term begins to dominate and the curve eventually bends down because Eq. (7) is a downward parabola if $K_{2}$ is negative. To avoid this bending-down phenomenon, one may further include the sixth-order term. Indeed, the fitting (using $K_{1}, K_{2}$, and $K_{3}$ as adjustable parameters) is very good in the entire region (blue curve). Nevertheless, this curve predicts a fast divergent trend in the high field region, as shown in Fig. 2.

To explain the saturation trend of our experimental data, we propose the following exponential convergence model:

$$
\delta n=\delta n_{\text {sat }}\left(1-\exp \left[-\left(\frac{E}{E_{S}}\right)^{2}\right]\right),
$$

where $\delta n_{s a t}$ stands for the saturated refractive index change and $E_{s}$ represents the saturation field. We used Eq. (8) to fit the experimental data leaving $\delta n_{s a t}$ and $E_{s}$ as adjustable parameters. The fitting with the experimental data is quite good in the entire region and, more importantly, it shows anticipated saturation trend in the high field region. For convenience, we call this convergence model as extended Kerr effect.

It is interesting to note that if we expand Eq. (8) into power series, we will obtain the $E^{2}$ term (Kerr effect) under weak field approximation and the Kerr constant can be written as follows:

$$
K=3 \delta n_{\text {sat }} /\left(\lambda E_{s}^{2}\right) .
$$

The fourth- and the sixth-order terms become increasingly important as $E$ increases. However, the inclusion up to the sixth-order term still does not lead to the saturation phenomenon in the high fields, as shown in Fig. 2.

The parameters obtained through fitting are $\delta n_{\text {sat }}$ $=0.038$ and $E_{s}=13.9 \mathrm{~V} / \mu \mathrm{m}$. The employed LC host has an intrinsic birefringence of 0.272 . Taking into account the LC concentration $(\sim 50 \%)$ and the factor of $1 / 3$ shown in Eq. (4), the obtained refractive index change is still $\sim 18 \%$ smaller than the ideal value, which is 0.045 . This difference could be attributed to following two factors: (1) a portion of LC is embedded in the polymer network, and some BPLCs are strongly anchored by the nanostructured double-twist cylinder boundaries so that they do not respond to the electric field, and (2) the relatively low clearing temperature $\left(\sim 60{ }^{\circ} \mathrm{C}\right)$ of the PS-BPLC so that the order parameter at the room temperature $\left(\sim 23{ }^{\circ} \mathrm{C}\right)$ is reduced accordingly. ${ }^{14}$

To validate the extended Kerr effect in a real display device, we incorporated Eq. (8) into our simulation program ${ }^{5}$ and tried to fit the experimental VT curve reported in Ref. 11 in an IPS cell with $5 \mu \mathrm{m}$ electrode width and $10 \mu \mathrm{m}$ electrode gap. Here, the induced birefringence $\left(\Delta n_{\text {ind }}\right)$ instead of the refractive index change $(\delta n)$ is used. Fitting is achieved by adjusting $\Delta n_{s a t}$ and $E_{s}$, where $\Delta n_{s a t}$ is the maximum induced birefringence. For comparison, the fitting result using 


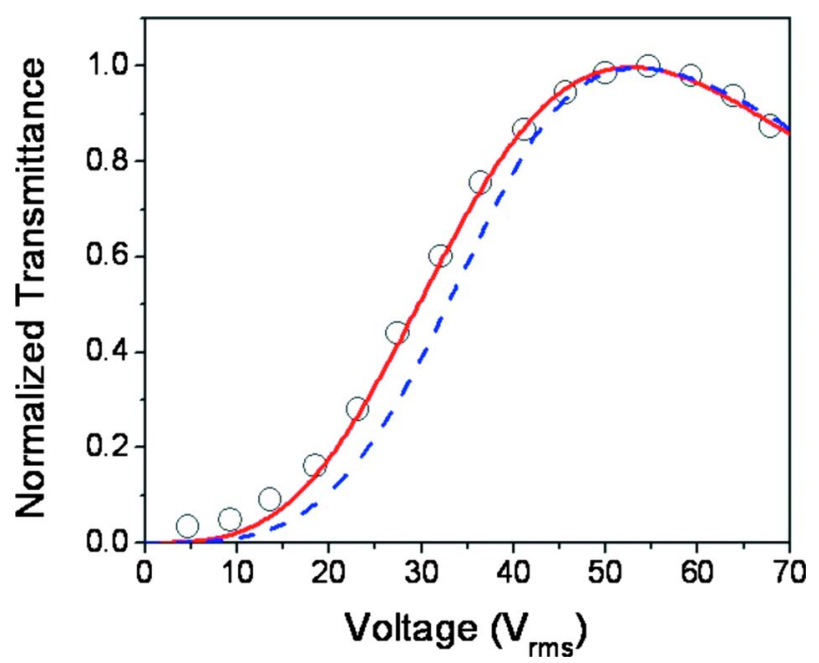

FIG. 3. (Color online) Model fittings with experimental data (circles; Ref. 11) of an IPS cell using the truncation model (dashed blue lines) and extended Kerr effect (solid red lines).

the truncation model [Eq. (6)] is also included in Fig. 3. The fitting using the extended Kerr effect (red curve) matches better with the experimental data than the truncation model (dashed blue lines). The slight mismatch below $20 \mathrm{~V}$ is due to the poor dark state of the experiment. Through fittings, we find; $\Delta n_{\text {sat }} \sim 0.068$ for both cases and $E_{s} \sim 2.02 \mathrm{~V} / \mu \mathrm{m}$ which implies to a Kerr constant $\mathrm{K} \sim 2.63 \times 10^{-8} \mathrm{~m} / \mathrm{V}^{2}$ in the low voltage region. On the other hand, the Kerr constant obtained using the truncation model is $1.62 \times 10^{-8} \mathrm{~m} / \mathrm{V}^{2}$. A larger Kerr constant leads to a higher transmittance in the low voltage region. Besides, the slower increase in induced birefringence at high voltage region results in a smoother transition near the peak transmittance.
In conclusion, the electro-optical properties of a PSBPLC are investigated. The experimental results reveal that the refractive index change follows the Kerr effect in the low field region but gradually saturates as the electric field keeps increasing. To explain this trend, an extended Kerr effect is proposed, which correctly predicts the saturation phenomenon in a test cell and fits well with the VT curve of an IPS display device. Potential application of this extended Kerr effect for optimizing PS-BPLC displays is foreseeable.

The authors are indebted to K. M. Chen for technical assistance and AFOSR for partial financial support under Contract No. FA95550-09-1-0170.

${ }^{1}$ H. Kikuchi, M. Yokota, Y. Hisakado, H. Yang, and T. Kajiyama, Nature Mater. 1, 64 (2002)

${ }^{2}$ Y. Hisakado, H. Kikuchi, T. Nagamura, and T. Kajiyama, Adv. Mater. 17, 96 (2005).

${ }^{3}$ S. W. Choi, S. I. Yamamoto, Y. Haseba, H. Higuchi, and H. Kikuchi, Appl. Phys. Lett. 92, 043119 (2008).

${ }^{4}$ Z. Ge, S. Gauza, M. Jiao, H. Xianyu, and S. T. Wu, Appl. Phys. Lett. 94, 101104 (2009).

${ }^{5}$ Z. Ge, L. Rao, S. Gauza, and S. T. Wu, J. Disp. Technol. 5, 250 (2009).

${ }^{6}$ L. Rao, Z. Ge, S. T. Wu, and S. H. Lee, Appl. Phys. Lett. 95, 231101 (2009).

${ }^{7}$ M. Jiao, Y. Li, and S. T. Wu, Appl. Phys. Lett. 96, 011102 (2010).

${ }^{8}$ K. M. Chen, S. Gauza, H. Xianyu, and S. T. Wu, J. Disp. Technol. 6, 49 (2010).

${ }^{9}$ J. Kerr, Philos. Mag. 50, 337 (1875).

${ }^{10}$ C. T. O'Konski, K. Yoshioka, and W. H. Orttung, J. Phys. Chem. 63, 1558 (1959).

${ }^{11}$ H. Kikuchi, Y. Haseba, S. Yamamoto, T. Iwata, and H. Higuchi, SID Int. Symp. Digest Tech. Papers 40, 578 (2009).

${ }^{12}$ J. Li, S. Gauza, and S. T. Wu, J. Appl. Phys. 96, 19 (2004).

${ }^{13}$ W. Jamroz, J. Karniewicz, and W. Kucharczyk, J. Phys. D 11, 2625 (1978).

${ }^{14}$ S. T. Wu, Phys. Rev. A 33, 1270 (1986). 